\title{
多铁性磁电复合薄膜
}

何泓材，林元华，南策文 ${ }^{*}$

清华大学材料科学与工程系, 新型陶瓷与精细工艺国家重点实验室, 北京 100084

*联系人, E-mail: cwnan@tsinghua.edu.cn

2007-12-05 收稿, 2008-02-20 接受

国家自然科学基金(批准号: 50621201, 10574078)和国家重点基础研究发展计划(编号: 2002CB613303)资助项目

摘要 多铁性材料由于其不但具有单一的铁性(如铁电性、铁磁性和铁弹性), 而且由于不 同铁性之间的耦合协同作用会产生新的磁电效应, 在换能器、传感器、存储器等高技术 领域具有巨大应用潜力. 其中, 磁电复合薄膜由于具有良好的微电子工艺兼容特性和良 好的性能, 并存在许多尚未解决的物理问题, 使得磁电复合薄膜的研究成为众多研究者 关注的热点. 从实验和理论两个方面介绍了目前多铁性磁电复合薄膜的研究现状. 综述 了 1-3 柱状结构、0-3 颗粒复合结构和 2-2 叠层结构等几种典型结构的磁电复合薄膜的实 验制备和性能, 同时介绍了用格林函数方法、相场模型和从头算方法等几种理论工具对 磁电复合薄膜模拟计算的结果. 最后, 指出了目前存在的问题和发展趋势.

关键词

多铁性

磁电效应

薄膜

复合材料 铁性(ferroic)材料(如铁电、铁磁材料)是一大类非 常重要的先进功能材料, 广泛应用于换能器、传感 器、敏感器等电子器件, 在传感、驱动、存储及智能 系统等高技术领域占主导地位. 在器件微型化、需求 多样化的现代生产生活中, 越来越迫切地需要同时 具备多种功能的材料, 多铁性(multiferroic)材料就是 其中的一类典型代表. 多铁性材料同时具备铁电、铁 磁等多种铁性，而且由于不同铁性之间的耦合作用 而具有磁电效应(magnetoelectric effect)等新的性能, 大大开拓了铁性材料的应用范围. 这类材料的发现, 为发展基于铁电-磁性集成效应的新型信息存储处理 以及磁电器件等技术提供了巨大的潜在应用潜力, 多铁性材料已成为当前国际上一个新的研究热点 ${ }^{[1]}$.

磁电效应是在材料外磁场作用下能够产生介电 极化(即正磁电效应: $P=\alpha H$ )或者在外电场作用下产 生磁极化的特性(即逆磁电效应: $M=\alpha E$ ), 既具有磁有 序和铁电有序两种有序结构共存, 同时两种有序结 构之间又存在一定形式耦合. 这里 $\alpha$ 是表征磁电材 料性能的磁电系数 (也可表示为 $E=\alpha_{E} H$ ). 磁电系数越 大, 表明磁电转换效率越高, 即磁有序与铁电有序之 间的耦合越强.
从组成上来看, 磁电材料可分为单相磁电材料 和复合磁电材料 ${ }^{[2,3]}$. 单相磁电材料是指本身具有磁 电效应的多铁性材料, 而复合磁电材料是指单相本 身并不具有磁电效应, 通过不同组成相之间的某种 耦合作用产生磁电效应的一类多相多铁性材料.

早在 1894 年居里预言了磁电效应的存在 ${ }^{[4]}$, 从 此掀开了研究磁电材料的序幕. 但直到 1961 年, 线 性磁电效应才首次在低温下的 $\mathrm{Cr}_{2} \mathrm{O}_{3}$ 晶体被观测到 [5,6]. 但是, 其磁电效应很弱. 随后, 在十多种不同系 列(如钇铁石榴石、硼酸物、稀土铁酸盐和磷酸盐)的 单相材料中 ${ }^{[7 \sim 9]}$, 也发现了弱的磁电效应, 但单相多 铁性磁电材料的研究一直处在缓慢的发展时期. 直 到近四年来, 对单相多铁性磁电化合物的研究又重 新掀起了热潮. 首先是在 $\mathrm{ReMnO}_{3}$ 这类稀土锰氧化物 中, 观察到其介电温谱在不同的外加磁场下表现出 了不同的行为, 表明磁场对介电性有调控作用 ${ }^{[10]}$. 最近, 关于 $\mathrm{BiFeO}_{3}$ 的薄膜材料的研究 ${ }^{[11,12]}$, 报道了室 温下具有良好的铁电性和铁磁性, 同时居里温度在 室温以上, 引起了研究者的广泛关注. 随后, 许多工 作致力于用掺杂等手段来提高这些单相多铁性化合 物的性能, 希望能够在室温下实现优异的铁电性和 
铁磁性共存, 以及磁电耦合效应 ${ }^{[13]}$. 目前, 以 $\mathrm{BiFeO}_{3}$ 为代表的多铁性化合物体系, 已形成一个世界范围 的单相多铁性磁电材料的研究热潮. 但目前, 单相多 铁性磁电化合物要么居里温度很低, 要么磁电效应 很微弱, 因而限制了它的实际应用. 寻找室温以上具 有强磁电耦合效应的单相多铁性化合物, 已成为多 铁性研究领域一个重要的方向.

在另一方面, 通过复合铁电/压电材料和磁致伸 缩材料, 以两相之间的应力/应变耦合传递可实现铁 电-铁磁之间的耦合, 这种由铁电/压电材料和磁性材 料复合在一起的磁电材料就是多铁性磁电复合材料 $[14,15]$. 自从 1974 年 Van Run等人 ${ }^{[16]}$ 报道 $\mathrm{BaTiO}_{3}$ $\mathrm{CoFe}_{2} \mathrm{O}_{4}$ 复合陶瓷的磁电系数比 $\mathrm{Cr}_{2} \mathrm{O}_{3}$ 大近两个数量 级以来, 人们开始关注复合磁电材料. 目前, 块体复 合磁电材料按其相组分, 可以大致分成陶瓷复合材 料 (例如, $\mathrm{BaTiO}_{3}$ - 铁氧体复合陶瓷 ${ }^{[17,18]}, \mathrm{Pb}(\mathrm{Zr}$,

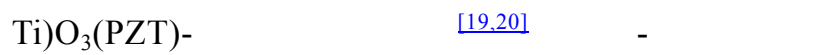
料(例如, PZT与琙镝铁合金 (Tb,Dy)Fe(Terfenol-D)的 黏结复合材料 ${ }^{[21 ~ 23]}$ )、高分子基复合材料(例如, Terfenol-D和PZT颗粒与高分子基体组成的三相复合材 料 $\left.{ }^{[24 ~ 27]}\right)$ 三类. 按照连通型结构, 又可以分为 $0-3$ 颗 粒复合、2-2 叠层结构、1-3 柱状结构等复合材料.

相比于块体磁电复合材料, 磁电复合薄膜材料 具有独特的优越性, 例如, (1) 复合材料中的铁电/压 电相与磁致伸缩相可以在纳米尺度上进行控制和调 节, 可在纳米尺度上研究磁电耦合机理; (2) 块体材 料中不同相之间的结合通过共烧或黏结的方式结合 在一起, 界面损耗是一个不容忽视的问题, 在薄膜中, 可实现原子尺度的结合，有效降低界面耦合损失; (3) 通过控制实验条件, 把晶格参数相近的不同相复合 在一起, 可获得高度择优取向甚至超晶格复合薄膜, 有利于研究磁电耦合的物理机理; (4) 多铁性磁电薄 膜可以用于制造集成的磁/电器件, 如微型传感器、

MEMS 器件、高密度的信息储存器件等.

近年来，随着薄膜制备技术的发展，使得制备优 质复杂结构的复合薄膜成为可能, 于是多铁性磁电 复合薄膜迅速成为众多研究者关注的热门方向. 特 别是最近三年来, 许多材料研究工作者从实验和理 论方面做了大量工作, 使得多铁性磁电复合薄膜的 研究取得了较大进展. 本文将从实验和理论两个方 面综述目前多铁性磁电复合薄膜的研究现状, 并分 析指出其存在的问题和挑战.

\section{1 磁电复合薄膜的实验研究}

实验上, 由于磁电复合薄膜涉及多种成分的复 合, 比较常见的是用激光脉冲沉积法(PLD)和溶胶凝胶旋涂法来制备, 这两种方法都可以方便地控制 复合薄膜的成分. 通过调节制备过程中的工艺参数, 可以得到不同结构的薄膜, 按照复合结构来分类, 我 们可以把磁电复合薄膜分为 1-3, 0-3, 2-2 等结构类型, 本节将按照不同结构类型评述磁电复合薄膜的实验 研究进展.

\subsection{1-3 型柱状复合磁电薄膜}

2004 年, Zheng等人 ${ }^{[28]}$ 报道了用激光脉冲沉积 (PLD)法在 $\mathrm{SrTiO}_{3}$ 单晶基片上制备 $\mathrm{BaTiO}_{3}-\mathrm{CoFe}_{2} \mathrm{O}_{4}$ 纳 米 1-3 结构复合薄膜, 同年 9 月, 又报道了相关结果 [29]，立刻引起了广泛关注. Zheng等人选用具有高压 电性能的 $\mathrm{BaTiO}_{3}$ 和具有高磁致伸缩性的 $\mathrm{CoFe}_{2} \mathrm{O}_{4}$ 的 复合陶瓷 $\left(0.65 \mathrm{BaTiO}_{3}-0.35 \mathrm{CoFe}_{2} \mathrm{O}_{4}\right)$ 为靶材, 用 PLD 方法在 $900^{\circ} \mathrm{C}$ 以上高温沉积, 在 $(001) \mathrm{SrTiO}_{3}$ 单晶基片 上, $\mathrm{BaTiO}_{3}-\mathrm{CoFe}_{2} \mathrm{O}_{4}$ 异质外延自组装产生相分离, 得 到的复合薄膜中 $\mathrm{CoFe}_{2} \mathrm{O}_{4}$ 形成纳米柱镶嵌在 $\mathrm{BaTiO}_{3}$ 基体中呈阵列分布, 形成了 1-3 结构的复合薄膜, 如 图 1 所示.

这种 $\mathrm{BaTiO}_{3}-\mathrm{CoFe}_{2} \mathrm{O}_{4}$ 复合薄膜的性能如图 2 所 示, 同时呈现了良好的铁电、压电和铁磁性能, 分别 与对应的单相 $\mathrm{BaTiO}_{3}$ 和 $\mathrm{CoFe}_{2} \mathrm{O}_{4}$ 性能相当. 实验发 现, 这种 1-3 结构的纳米复合薄膜在铁电居里温度附 近, 其磁化强度随温度的变化出现了异常变化, 如图 2(c)所示, 表明这种复合薄膜具有磁电耦合效应, 即 在铁电居里温度附近, $\mathrm{BaTiO}_{3}$ 从铁电到顺电相转变通 过弹性相互作用影响到 $\mathrm{CoFe}_{2} \mathrm{O}_{4}$ 的磁化强度. 虽然这 只是间接、定性地证实薄膜中的磁电耦合效应, 没有 直接测量到复合薄膜的磁电耦合性能, 但这是第一 次在复合薄膜中发现磁电耦合效应的报道.

随后, 该课题组又用同样的方法在 $\mathrm{SrTiO}_{3}$ 单晶 基片上制备得到了 $\mathrm{BiFeO}_{3}-\mathrm{CoFe}_{2} \mathrm{O}_{4}$ 复合 1-3 结构 ${ }^{[30]}$, 并就这个体系开展了一系列工作 ${ }^{[31,32]}$. 重要的是, 他 们近来在这个结构中, 利用扫描探针显微镜观察到 了由施加电场诱导磁化翻转的现象(图 3), 由此表明 在这种 1-3 柱状纳米结构中存在着磁电耦合效应, 从 而给出了薄膜中磁电耦合效应的直接证据. 他们首 先通过磁力显微镜(MFM)观察到了电极化之后的复 合薄膜出现了磁畴翻转, 证明了两个复合相之间存在 

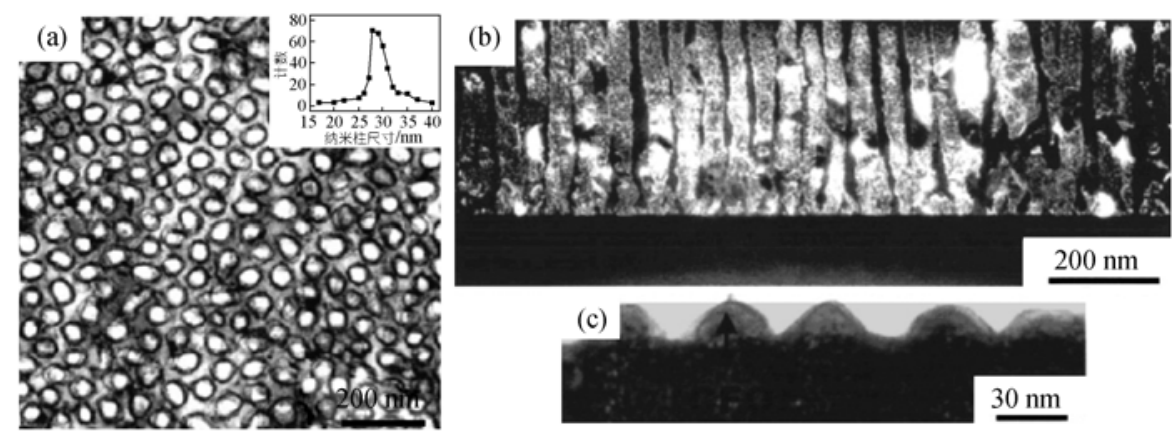

图1 在 $\mathrm{SrTiO}_{3}$ 单晶基片外延生长的 $\mathrm{BaTiO}_{3}-\mathrm{CoFe}_{2} \mathrm{O}_{4}$ 纳米 1-3 复合薄膜的形貌 $[28,29]$

(a) TEM 平视形貌像; (b) TEM 截面暗场像; (c) TEM 截面明场像, 显示了 $\mathrm{CoFe}_{2} \mathrm{O}_{4}$ 纳米柱露头
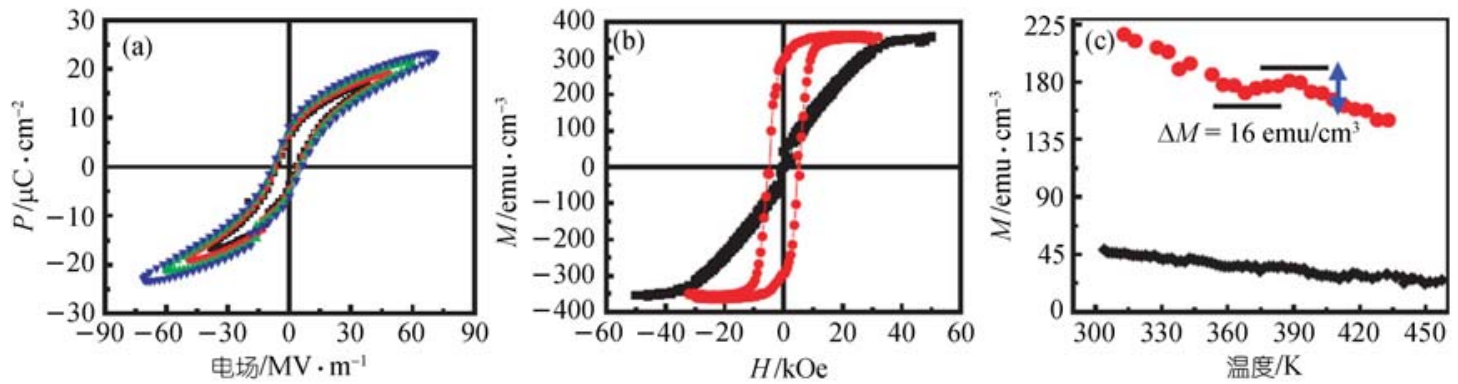

图2 $\mathrm{BaTiO}_{3}-\mathrm{CoFe}_{2} \mathrm{O}_{4}$ 复合薄膜的性能

(a) 电滞回线; (b) 磁带回线 $\left(1 \mathrm{Oe}=79.58 \mathrm{~A} / \mathrm{m}\right.$ ); (c) 磁化强度随温度的变化 ${ }^{[28]}$
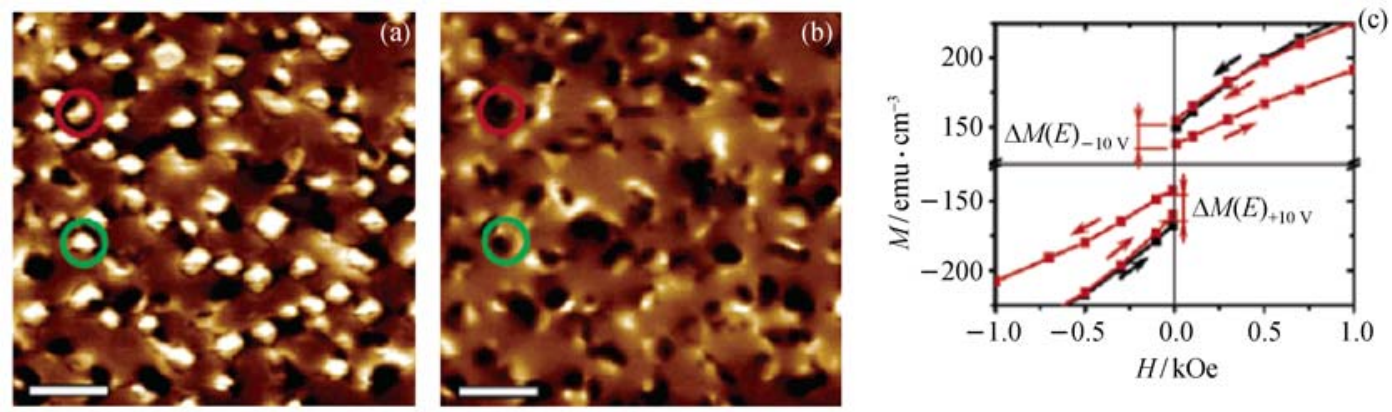

图3 复合薄膜中电场诱导磁畴翻转(a)(b)及磁化强度变化(c) $)^{[30]}$

着耦合. 同时还利用 SQUID 测得了电极化之后复合 薄膜磁化强度的变化(图 3(c)), 并由此估算出复合薄 膜的磁电敏感系数 $\alpha_{33}=\Delta M / \Delta E \approx 1.0 \times 10^{-2} \mathrm{G} \cdot \mathrm{cm} / \mathrm{V}$.

进一步, 通过选择不同取向的 $\mathrm{SrTiO}_{3}$ 单晶基片 和控制复合薄膜两相的体积分数, 可以对复合薄膜 的形貌进行调控, 得到不同形貌和结构的 1-3 柱状复 合薄膜 ${ }^{[32]}$, 如图 4 所示. Zheng等人从机理上对其生 长过程进行了研究, 认为这是由于晶体表面能的各 向异性, 不同比例的钙钛矿结构 $\mathrm{BiFeO}_{3}$ 和尖晶石结 构 $\mathrm{CoFe}_{2} \mathrm{O}_{4}$ 在不同取向的单晶 $\mathrm{SrTiO}_{3}$ 基片上外延生长 自组装而造成的不同结构.
从另一方面, Levin等人 ${ }^{[33]}$ 也生长了类似结构和 形貌的 $x \mathrm{CoFe}_{2} \mathrm{O}_{4}-(1-x) \mathrm{PbTiO}_{3}$ 复合薄膜. 但他们给出 了不同解释, 认为不同的基片使薄膜处于不同的应 力状态, 从而使最终的纳米复合薄膜具有不同的结 构和形貌. 为了验证他们的解释, 他们利用相场方法 考虑不同应力状态, 模拟了复合薄膜的生长, 得到与 实验观察类似的结果.

虽然 1-3 柱状纳米结构复合薄膜表现出了具有较 强磁电耦合的迹象, 但这种 1-3 柱状纳米结构复合薄 膜的生长需要比较苛刻的条件, 如相当高的生长温 度 (大于 $900^{\circ} \mathrm{C}$ ), 其纳米柱状阵列生长也是不易控制 


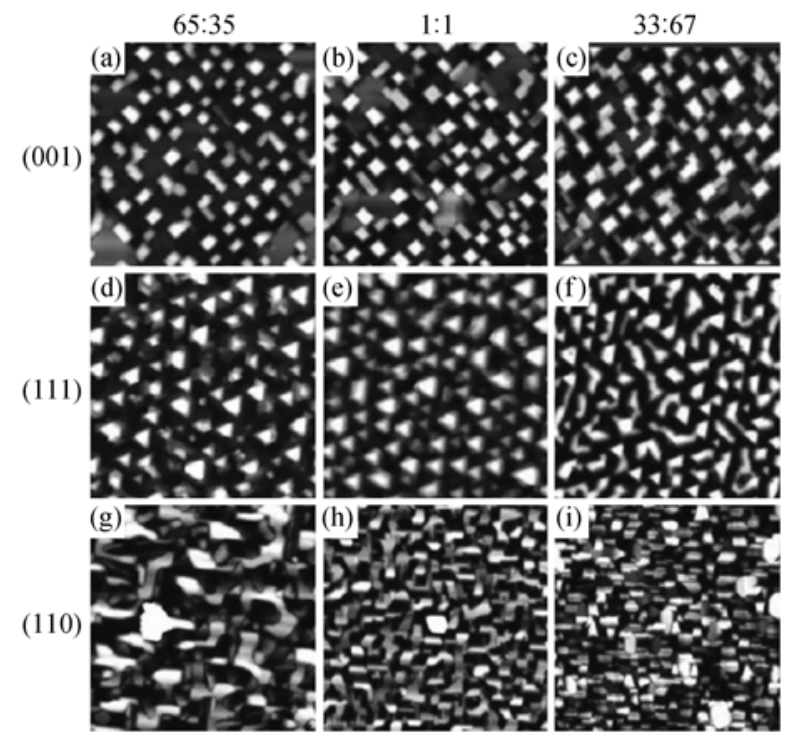

图4 不同成分的 $\mathrm{BiFeO}_{3}-\mathrm{CoFe}_{2} \mathrm{O}_{4}$ 复合薄膜 ${ }^{[32]}$

的. 另一方面, 由于电阻特性较差的磁性相贯穿整个 薄膜导致薄膜漏导太大, 不易直接观测到正磁电效 应，从而将使其应用受到限制.

\subsection{0 -3 型颗粒复合磁电薄膜}

0-3 型复合磁电薄膜就是一种颗粒复合多晶薄膜. 南京大学 $W a n$ 等人 ${ }^{[34]}$ 采用溶胶-凝胶法在 $\mathrm{Pt} / \mathrm{Ti} / \mathrm{SiO}_{2} /$ $\mathrm{Si}$ 基片上把 $\mathrm{CoFe}_{2} \mathrm{O}_{4}$ 和 $\mathrm{Pb}(\mathrm{Zr}, \mathrm{Ti}) \mathrm{O}_{3}$ 前驱溶胶交替旋涂, 然后在 $650^{\circ} \mathrm{C}$ 退火 $6 \mathrm{~min}$, 两相在退火过程中产生分 离重组, 得到了一种疑似颗粒复合薄膜, 如图 5 所示. 从薄膜表面形貌来看, 似乎 $\mathrm{CoFe}_{2} \mathrm{O}_{4}$ 以颗粒状分散在 PZT 基体中, 形成了 0-3 结构的多晶复合薄膜. 测量 表明这种薄膜同时表现出较好的铁磁、铁电响应, 并 测量到磁电复合薄膜的磁电系数. 近来, Zhong等人 ${ }^{[35]}$ 用类似的化学方法得到了 $x \mathrm{Bi}_{3.15} \mathrm{Nd}_{0.85} \mathrm{Ti}_{3} \mathrm{O}_{12}$ $(1-x) \mathrm{CoFe}_{2} \mathrm{O}_{4}$ 体系的颗粒复合薄膜, 也观察到较好的 铁磁、铁电性能, 并对薄膜的磁电系数进行直接测量.

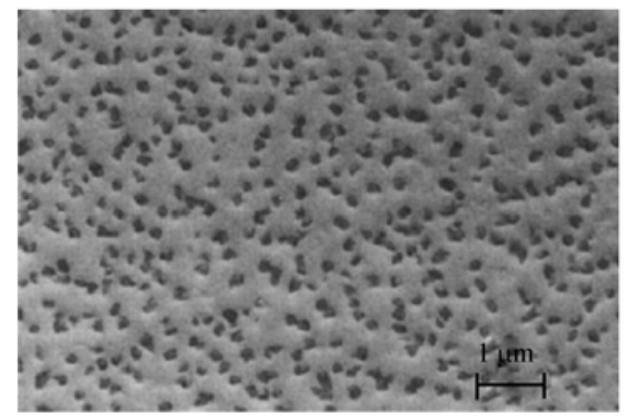

图5 溶胶-凝胶法制备磁电PZT/CFO复合薄膜的 SEM形貌图 [34]
最近，Ryu等人 ${ }^{[36]}$ 用PLD方法在掺杂 $0.5 \% \mathrm{Nb}$ 的 (110) $\mathrm{SrTiO}_{3}$ 基片上制备了 $\mathrm{PZT}-\mathrm{NiFe}_{2} \mathrm{O}_{4}$ 复合薄膜. $\mathrm{NiFe}_{2} \mathrm{O}_{4}$ 以纳米颗粒随机分布在PZT基体中, 如图 6 所示. 这种薄膜同时表现出较好的铁磁、铁电性能. 他们用不同的电压对复合薄膜进行极化, 测量了极 化之后的复合薄膜的磁电系数, 结果表明在大约 0.8 $\mathrm{MV} / \mathrm{cm}$ 的电场作用下磁电系数达到饱和. 然后用 +0.8 和 $-0.8 \mathrm{MV} / \mathrm{cm}$ 的电场对复合薄膜极化之后进行 测量, 观察到了磁电系数随偏压静磁场的变化关系, 如图 7 所示. 随着静磁场的增加, 磁电系数先增大, 到 一个极大值之后开始减小, 而磁电系数 $\alpha_{\mathrm{E} 31}$ 相比于 $\alpha_{\mathrm{E} 33}$ 在更小的静磁场就达到极大值, 这些变化规律与 块体磁电复合陶瓷的规律是类似的 ${ }^{[37]}$. 但在这种 PZT-NiFe ${ }_{2} \mathrm{O}_{4}$ 复合薄膜中, $\alpha_{\mathrm{E} 31}$ 最大约为 $4 \mathrm{mV} /$ $(\mathrm{cm} \cdot \mathrm{Oe})(1 \mathrm{Oe}=79.58 \mathrm{~A} / \mathrm{m}), \alpha_{\mathrm{E} 33}$ 最大约为 $16 \mathrm{mV} /$ $(\mathrm{cm} \cdot \mathrm{Oe})$, 明显小于块体磁电复合陶瓷的磁电系数. 一般认为在复合薄膜中基片对薄膜的约束效应导致 了较小的磁电系数.
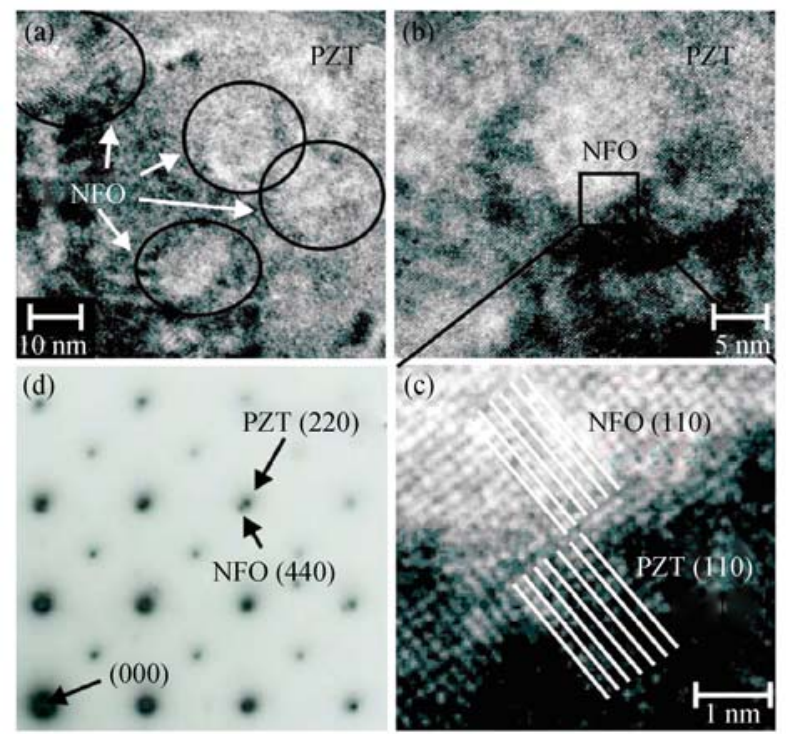

图6 PZT-NFO复合薄膜的表面形貌以及晶格衍射图 $[36]$ (a) 低放大倍数 HRTEM 表面形貌; (b) PZT-NFO 边界附近的 HRTEM 形貌; (c) 图(b)中所选区域的晶格像; (d) 复合薄膜选区 电子衍射图

最近, Liu等人 ${ }^{[38]}$ 用一种改进的溶胶-凝胶方法也 得到了颗粒复合的多晶薄膜. 他们先将磁性相 CFO 和铁电相 P Z T 的溶胶进行充分混合, 然后在 $\mathrm{Ru} / \mathrm{SiO}_{2} / \mathrm{Si}$ 基片上通过旋涂工艺得到薄膜, 从而得到 了纳米尺度复合的CFO-PZT多晶复合薄膜, 其中两 相颗粒粒径 5 10 nm, 如图 8 所示. 在这种薄膜中, 
(a)

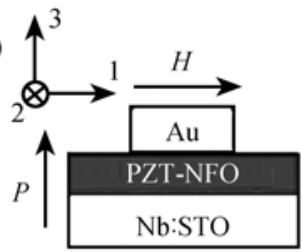

(b)
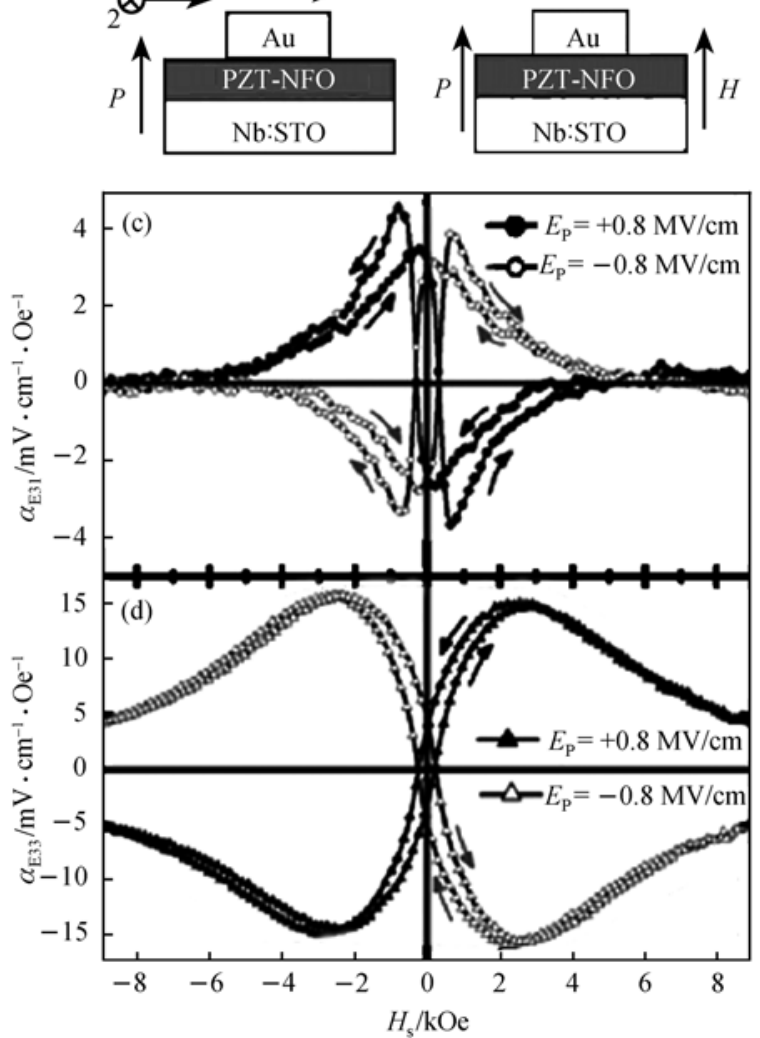

图7 磁电系数 $\alpha_{\mathrm{E} 31}(\mathrm{a})$ 和 $\alpha_{\mathrm{E} 33}(\mathrm{~b})$ 测量示意图以及 $\alpha_{\mathrm{E} 31}(\mathrm{c})$ 和 $\alpha_{\mathrm{E} 33}(\mathrm{~d})$ 测量结果
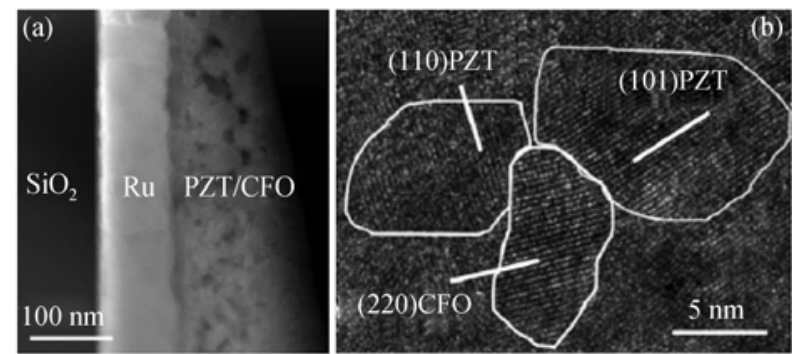

图8 多铁性CFO-PZT纳米复合薄膜的截面STEM(a) 和HRTEM(b)照片 ${ }^{[38]}$

他们观察到了较强的磁电耦合效应，通过测量不同 磁场环境中的电滞回线观察到了磁场诱导电极化现 象. 如图 9 所示, 当垂直于膜面加 $1200 \mathrm{Oe}$ 的磁场时, 复合薄膜的剩余极化值减小了 $22 \%$ ，从而认为这种复 合薄膜中具有较强的磁电耦合. 但是, 从其电滞回线 来看, 该薄膜漏导较大, 因而电滞回线的变化是否是 反映了真正的磁电耦合效应，还值得商榷.

对于 0-3 颗粒复合薄膜, 也需要注意漏导的问题.

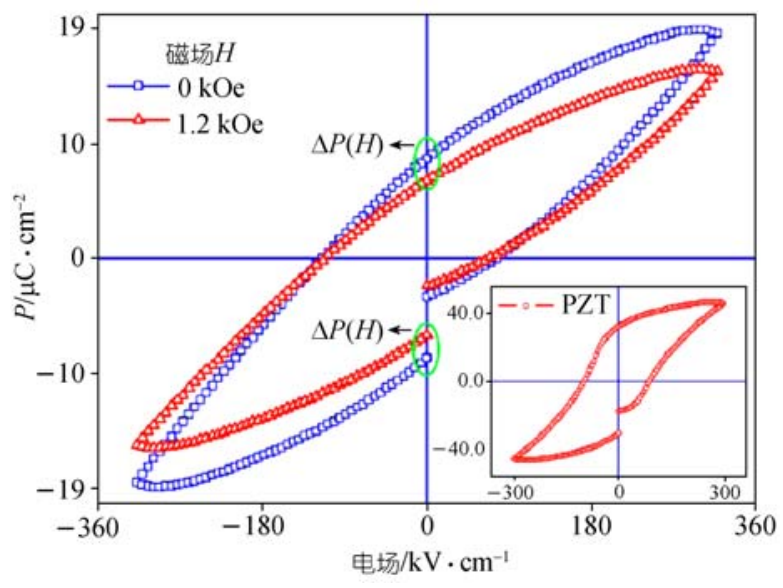

图9 多铁性CFO-PZT纳米复合薄膜的电滞回线

如若磁性相颗粒发生渗流而连通在一起，则低电阻 的磁性相会引起薄膜的整体漏导, 导致观测到正磁 电效应有误，甚至观测不到正磁电效应.

\subsection{2-2 型叠层复合磁电薄膜}

将铁电、铁磁两相物质一层一层地沉积在基片上 就可以得到叠层结构的磁电复合薄膜. 由于叠层结 构薄膜受基片应力约束的影响较大，使得一段时间 内，人们对这种结构并不看好. 但是由于这种结构的 实用性以及制备工艺易于控制, 又使得人们对其进 行了不断的努力尝试和探索.

Takeuchi等人 ${ }^{[39,40]}$ 用改进的PLD方法，通过在沉 积腔中添加一个可以匀速移动的挡板实现对厚度的 调制, 得到了 $\mathrm{BaTiO}_{3}$ 和 $\mathrm{CoFe}_{2} \mathrm{O}_{4}$ 的成分梯度复合薄膜, 如图 10(a)所示, 在这种薄膜中, 两相成分呈连续变 化. 通过对微区性能的测试，可以方便、高效地对两 相成分对性能的影响进行研究. 如图 10(b)所示, 在 成分 $(\mathrm{BTO})_{0.5}-(\mathrm{CFO})_{0.5}$ 处, 复合薄膜在呈现磁性的同 时可以具有高达 120 的介电常数. 但是由于微区性能 的测试还存在困难, 所以这种薄膜没有能够得到磁 电测试结果.

更常见的 2-2 结构的磁电复合薄膜是用 PLD 制 备外延薄膜, 或者用化学溶液旋涂法制备得到叠层 多晶薄膜, 由这两种方法都能够很方便地制备优质 的磁电复合薄膜. 相对 1-3 和 0-3 型复合薄膜而言, 2-2 结构中低电阻的磁性层在面外方向被绝缘的铁电 层所隔离, 因而 2-2 结构可完全消除漏导问题.

$\mathrm{He}$ 等人 $[41,42]$ 利用化学溶液旋涂法, 将PZT和 $\mathrm{Co}_{1-x} \mathrm{Zn}_{x} \mathrm{FeO}_{3}(\mathrm{CZFO})$ 前驱溶胶依次旋涂在 $\mathrm{Pt} / \mathrm{Ti} / \mathrm{SiO}_{2} /$ 
(a)
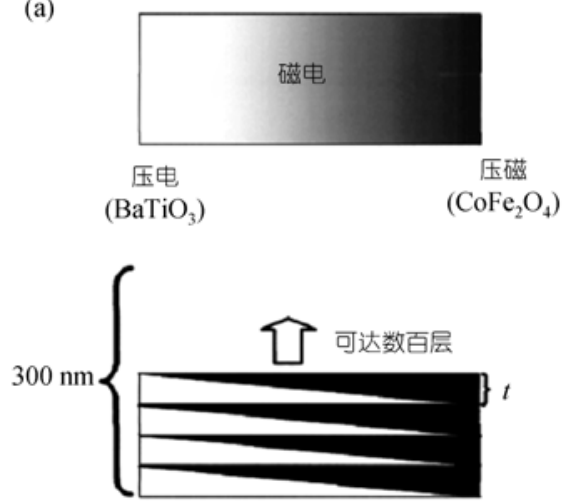

(b)

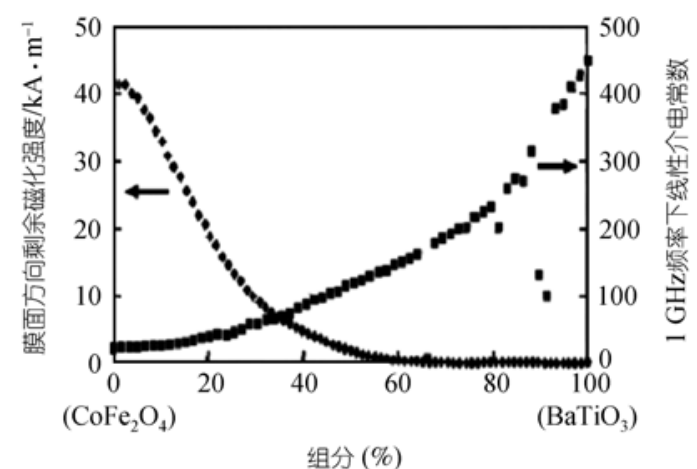

组分 $(\%)$

图 $10 \mathrm{BaTiO}_{3}$ 和 $\mathrm{CoFe}_{2} \mathrm{O}_{4}$ 的成分梯度复合薄膜示意图(a)以及性能随成分的变化(b)

$\mathrm{Si}$ 基片上，可以方便地得到多层结构的复合薄膜. 同 样按照两相不同的沉积顺序可以得到两种多层结构 (如图 11(a)和(b)), 即基片/PZT/CZFO/PZT/CZFO(简称 为 $\mathrm{PCPC}$ )和基片 $/ \mathrm{CZFO} / \mathrm{PZT} / \mathrm{CZFO} / \mathrm{PZT}$ (简称为 $\mathrm{CPCP}$ ). 其电学性能如图 11(c)和(d)所示, 结果表明, 相比于 纯的PZT薄膜, 由于复合薄膜中的铁氧体相具有较低 的电阻率, 复合薄膜具有更大的漏电流; 但是两种结 构的复合薄膜之间的漏电流特性没有体现出明显的 差别, 对沉积顺序不敏感. 而同样由于顺电相的钴铁 氧体层的存在, 复合薄膜的铁电性相比单相的PZT要 弱一些. 复合薄膜的磁性如图 11(e)和(f)所示, 结果 显示其具有良好的铁磁性能. 由于非铁磁的PZT层的 存在, 复合薄膜的磁化强度明显低于单相的铁氧体. 而同时，由于复合薄膜中有更多的应力，根据压磁效 应，复合薄膜也具有了明显低于单相铁氧体的矫顽 力 $[41,42]$.

用溶胶-凝胶法制备得到的复合薄膜显示了良好 的、同时共存的铁电、铁磁性能, 分别与单相的PZT 的铁电性能和铁氧体的铁磁性能基本相当. 进一步 研究复合结构对于复合薄膜性能的影响表明 ${ }^{[41]}$, 在 保持复合薄膜总厚度基本一致的前提下，复合薄膜 的层数不同，复合薄膜的性能也体现出相应的差异. 随着铁氧体层远离基片, 薄膜的饱和磁化强度逐渐 增大, 这是由于铁氧体层远离基片，薄膜内部的应力 释放引起的. 而对于铁电性能, 没有明显的差异.

类似的，用PLD方法也可以方便地得到叠层结 构复合薄膜. 最近, Deng等人 ${ }^{[43]}$ 在 $\mathrm{SrTiO}_{3}$ 单晶基片上 外延得到了 $\mathrm{BaTiO}_{3} / \mathrm{NiFe}_{2} \mathrm{O}_{4}$ 两层结构的薄膜, 如图 12 所示, 这种薄膜具有很好的外延特性, 而且两层之间 界面清晰. 这种薄膜也同时显示出了良好的、与单相
材料相当的铁电和铁磁性能, 特别值得注意的是, 他 们还在这种复合薄膜中直接观察到了显著的磁电响 应, 如图13(a)所示. 通过对薄膜施加交变方波磁场激 励，复合薄膜也出现了相应的方波响应电压信号，而 这是两种单相薄膜所不具备的, 这直观地对磁电响 应信号给出了明晰的证据. 同时, 该响应信号还随交 变磁场的幅值呈线性变化, 其斜率就是磁电电压系 数. 借用块体磁电材料中磁电电压系数的定义: $\alpha_{\mathrm{E}}=\delta V /(t \cdot \delta H)$ (其中 $t$ 为复合薄膜的厚度), 由图 13(b)中线性关系的斜率可以得到该复合薄膜在垂直 于薄膜方向的磁电电压系数为 $12 \mathrm{mV} /(\mathrm{cm} \cdot \mathrm{Oe})$. 考虑 到薄膜受到了较强的基片应力约束, 这样磁电耦合 效应已是十分可观

Zhou等人 ${ }^{[44,45]}$, Ryu等人 ${ }^{[46]}$ 和Ziese等人 ${ }^{[47]}$ 也都 用 PLD方法做了类似的工作。Zhou等人得到的 $\mathrm{CoFe}_{2} \mathrm{O}_{4} / \mathrm{PZT}$ 两层结构薄膜 ${ }^{[44,45]}$, Ryu等人制备的 $\mathrm{PZT} / \mathrm{Ni}_{0.8} \mathrm{Zn}_{0.2} \mathrm{Fe}_{2} \mathrm{O}_{4}$ 多层结构薄膜 ${ }^{[46]}, \mathrm{Ziese}$ 等人制备 的 $\mathrm{Fe}_{3} \mathrm{O}_{4} / \mathrm{BaTiO}_{3}$ 两层结构薄膜 ${ }^{[47]}$, 都有着良好的薄 膜质量及铁电与铁磁共存的性能, 同时还得到了磁 电系数为 $15 \sim 30 \mathrm{mV} /(\mathrm{cm} \cdot \mathrm{Oe})$ 的磁电耦合性能.

Murugavel等人 ${ }^{[48,49]}$ 和Singh等人 ${ }^{[50,51]}$ 先后报道 了用PLD方法制备的超晶格结构复合磁电薄膜，涉及 $\mathrm{Pr}_{0.85} \mathrm{Ca}_{0.15} \mathrm{MnO}_{3} / \mathrm{Ba}_{0.6} \mathrm{Sr}_{0.4} \mathrm{TiO}_{3}{ }^{[48,49]}$ 和 $\mathrm{La}_{0.7} \mathrm{Ca}_{0.3} \mathrm{MnO}_{3} /$ $\mathrm{BaTiO}_{3}{ }^{[50,51]}$ 两个体系。在 $\mathrm{SrTiO}_{3}$ 单晶基片上的 $\mathrm{Pr}_{0.85} \mathrm{Ca}_{0.15} \mathrm{MnO}_{3} / \mathrm{Ba}_{0.6} \mathrm{Sr}_{0.4} \mathrm{TiO}_{3}$ 和 $\mathrm{La}_{0.7} \mathrm{Ca}_{0.3} \mathrm{MnO}_{3} / \mathrm{Ba}-$ $\mathrm{TiO}_{3}$ 超晶格多层薄膜都显示了良好的介电性和铁磁 性, 并显示出磁阻效应和磁电容效应.

\section{4 准 2-2 型磁电薄膜}

之所以称为准 2-2 型, 是为了与前一节中的 2-2 型叠层复合薄膜区别开. 这种准 2-2 型复合实际上是 

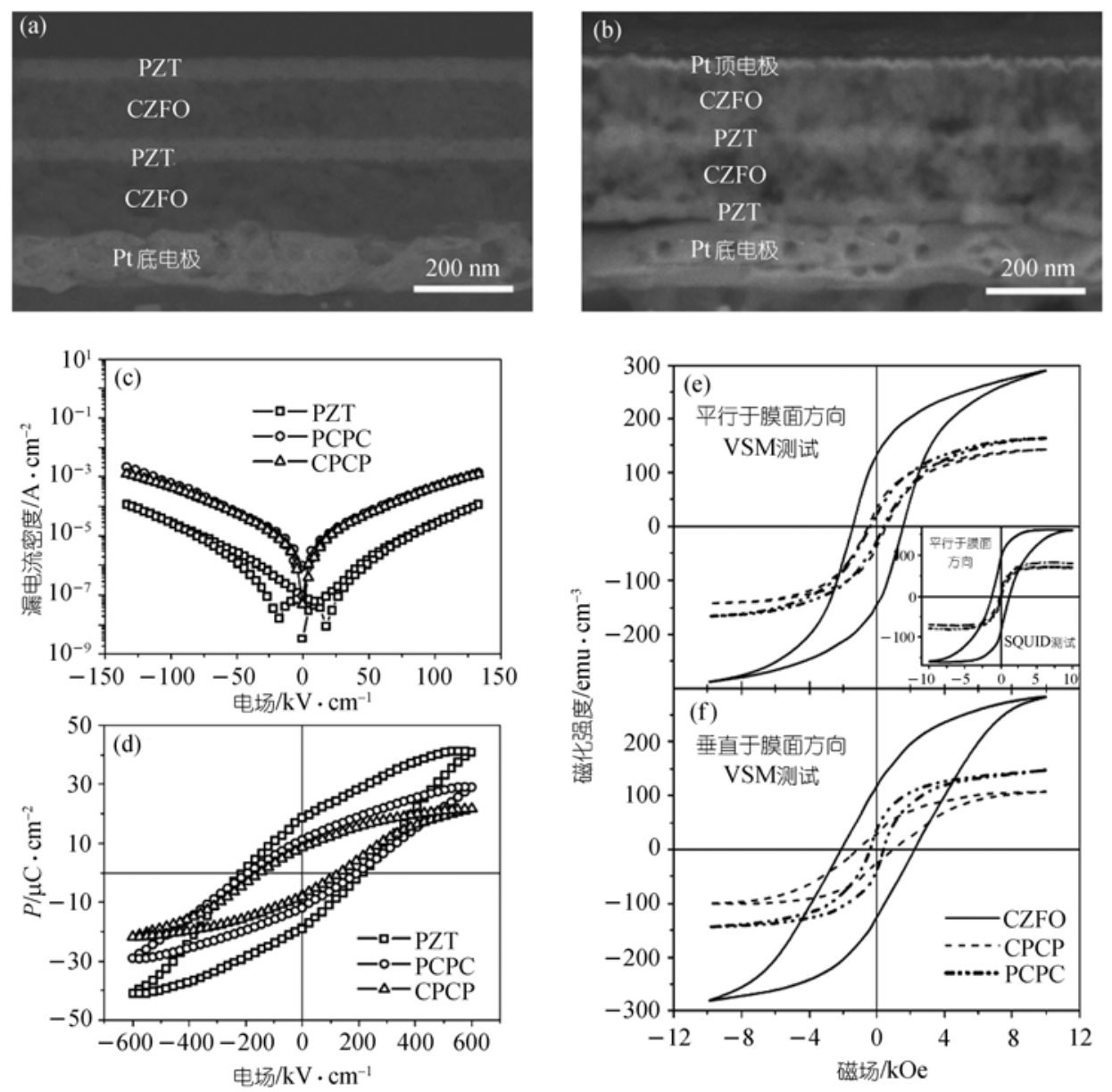

图11 CPCP和PCPC复合薄膜的显微结构(a)(b)以及电性能(c)(d)和磁性能(e)(f) ${ }^{[411}$
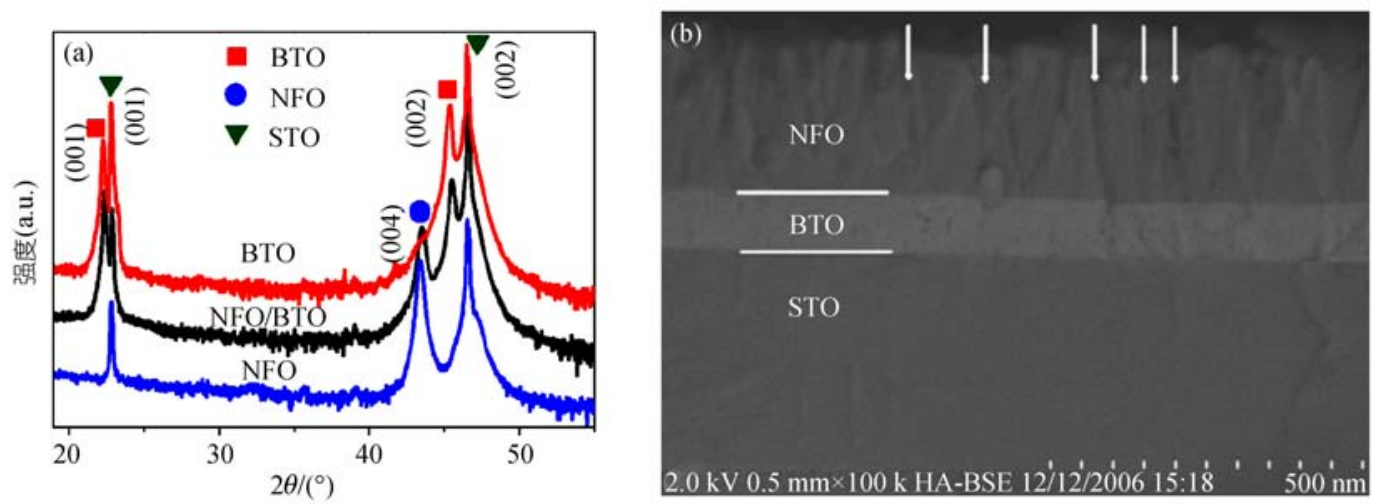

图12 $\mathrm{PLD}$ 制备 $\mathrm{BaTiO}_{3} / \mathrm{NiFe}_{2} \mathrm{O}_{4}$ 两层结构复合薄膜的XRD图谱(a)和截面SEM形貌(b) ${ }^{[43]}$

块体与薄膜的复合, 在铁性块体基片上直接沉积另 外一种铁性薄膜, 例如, 在压电基片上沉积磁性薄膜, 或者是在磁性基片上沉积压电薄膜.

Stein等人 ${ }^{[52]}$ 在 $100 \mu \mathrm{m}$ 厚的PMN-PT单晶基片两 边沉积 $4 \mu \mathrm{m}$ 厚的 $\mathrm{Tb}_{40} \mathrm{Fe}_{60} / \mathrm{Fe}_{50} \mathrm{Co}_{50}$ 合金厚膜(如图 14(a)所示结构示意图), 性能测试表明, 磁电电压跟 交流磁场幅值之间有很好的线性关系(图14(b)), 并在 这种薄膜中观测到磁电系数为 $24 \mathrm{mV} /(\mathrm{cm} \cdot \mathrm{Oe})$.

Eerenstein等人 ${ }^{[53]}$ 在 $(100) \mathrm{BaTiO}_{3}$ 铁电单晶基片 上用PLD方法沉积了 $40 \mathrm{~nm}$ 厚的 $\mathrm{La}_{0.67} \mathrm{Sr}_{0.33} \mathrm{MnO}_{3}$ 

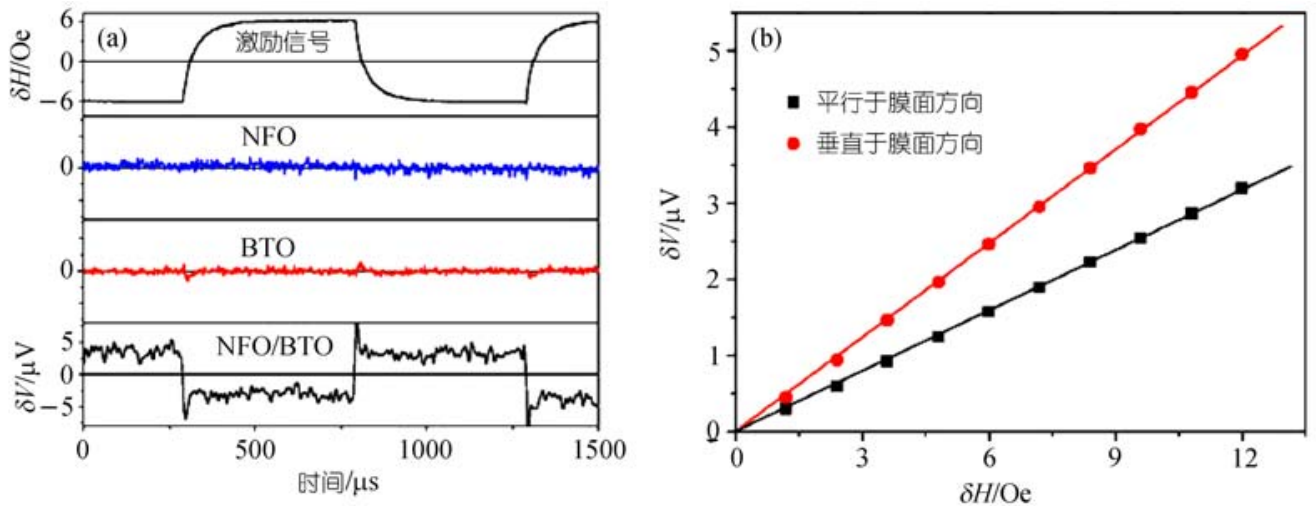

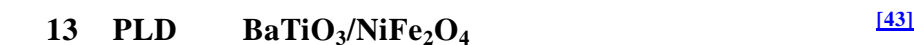

(a) 薄膜在平行于膜面方向方波交流磁场作用下的磁电响应信号; (b) 诱导磁电电压隧交流磁场的变化情况
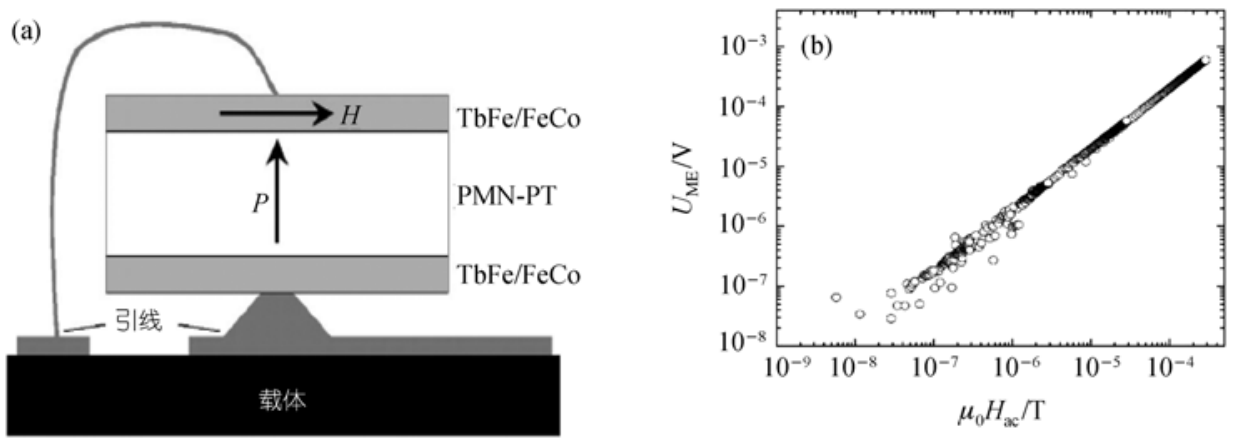

图14 磁电复合材料结构示意图(a)及磁电电压随交流磁场幅值变化关系(b)

(LSMO)薄膜. 这个结构在电场作用下, $\mathrm{BaTiO}_{3}$ 单晶 基片内的电畴发生变化, 从而产生形变. 形变作用到 薄膜上, 导致 LSMO 薄膜磁结构发生变化, 如图 15 所示, 从而实现由电场控制磁极化的逆磁电效应.

$\mathrm{Wu}$ 等人 ${ }^{[54,55]}$ 在 $\mathrm{La}_{1.2} \mathrm{Sr}_{1.8} \mathrm{Mn}_{2} \mathrm{O}_{7}$ 单晶基片上外延 $110 \mathrm{~nm}$ 厚的PZT薄膜, 性能测试表明, 该结构在不同

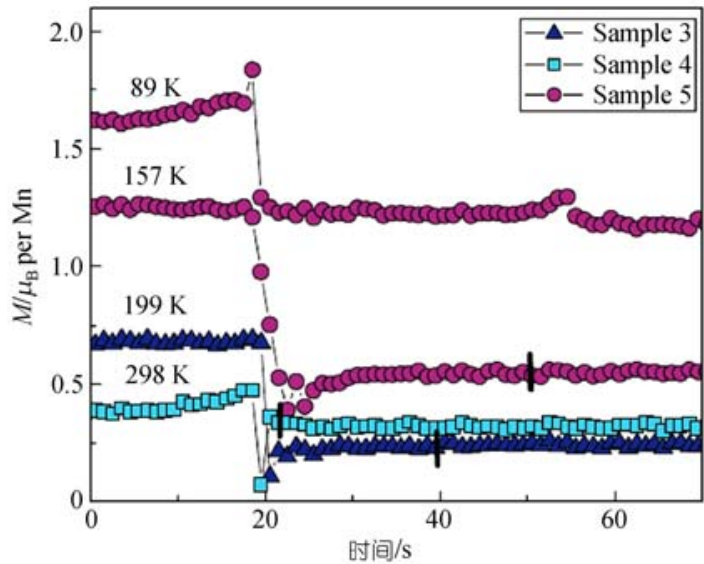

图15 薄膜中电场作用引起的磁开关现象 53$]$

所加电场: $4 \mathrm{kV} / \mathrm{cm}$ (Sample 3), $10 \mathrm{kV} / \mathrm{cm}$ (Sample 4), $6 \mathrm{kV} / \mathrm{cm}$ (Sample 5)
温度下具有不同的磁电耦合性能(图 16), 接近 $\mathrm{La}_{1.2}$ $\mathrm{Sr}_{1.8} \mathrm{Mn}_{2} \mathrm{O}_{7}$ 居里温度的 $120 \mathrm{~K}$ 时观察到较大的磁致伸 缩和磁电效应, 磁电系数最大可达 $600 \mathrm{mV} /(\mathrm{cm} \cdot \mathrm{Oe})$. 由于具有很好的界面结合性, 与唯象热力学理论预 测结果非常接近, 为理论值的 $87 \%$, 这和块体关于 2-2 型结构复合的理论预测是一致的.

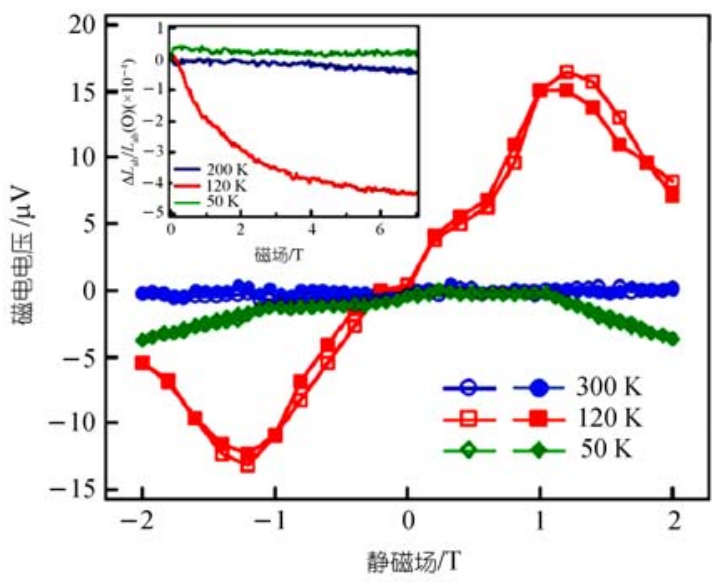

图16 不同温度磁电电压对静磁场的响应 插入图为磁致伸缩对静磁场的响应 
与现有微电子工艺的兼容是磁电薄膜材料应用 的必备条件. 虽然这种准 2-2 结构表现出了较好的磁 电耦合特性, 但是由于这类特殊的磁电薄膜对基片 有着特殊的要求, 需要基片为铁电、铁磁中的一种, 这大大限制了这类薄膜材料的应用.

\section{2 磁电复合薄膜的理论研究}

自 2004 年Zheng等人 ${ }^{[28]}$ 报道了有关 $\mathrm{BaTiO}_{3}$ 和 $\mathrm{CoFe}_{2} \mathrm{O}_{4}$ 磁电复合薄膜的实验结果之后, 关于磁电复 合薄膜中耦合机理的理论工作成为人们关注的一个 热点.

首先是 $\mathrm{Nan}$ 等人 [56]利用格林函数方法对 $\mathrm{BaTiO}_{3}$ 和 $\mathrm{CoFe}_{2} \mathrm{O}_{4}$ 磁电复合薄膜中的磁场诱导电极化(MIEP) 进行了理论模拟和计算. 通过对 1-3 和 2-2 两种结构 复合薄膜中的磁电耦合效应进行比较计算, 结果表 明 1-3 结构薄膜的MIEP较大(图 17), 而完全受基片约 束的 2-2 结构薄膜的MIEP十分微弱, 这与Zheng等人 ${ }^{[28]}$ 的实验结果在定性上是相符的. 两种结构薄膜之 间的这种差异, 主要是由于考虑了宏观的刚性基片 对 2-2 结构薄膜施加了完全的约束作用. 对于块

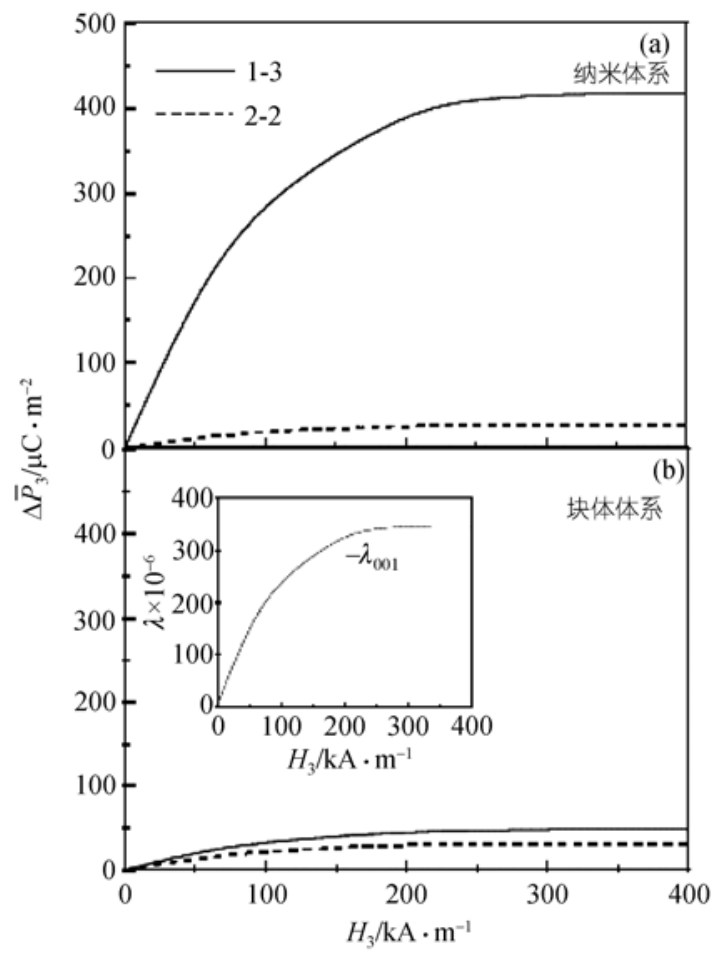

图17 室温下 $\mathrm{BaTiO}_{3} / \mathrm{CoFe}_{2} \mathrm{O}_{4}$ 复合材料磁场诱导极化强 度随磁场强度变化关系 ${ }^{[56]}$

(a) 纳米复合薄膜结构; (b) 块体复合结构, 插图为 $\mathrm{CoFe}_{2} \mathrm{O}_{4}$ 磁致 伸缩曲线
体复合磁电材料(图 17(b)), 1-3 结构和 2-2 结构差别不 大. 但是对于纳米复合薄膜(图 17(a)), 1-3 结构的磁 场诱导电极化远大于 2-2 结构, 且 1-3 结构中的 MIEP 随磁场的增加呈非线性增加, 变化趋势与其中磁性 相的磁致伸缩曲线基本一样，在磁场达到 $240 \mathrm{kA} / \mathrm{m}$ 以上时, CFO 的磁致伸缩达到饱和, 此时 MIEP 随磁 场的增加基本不变, 也达到了饱和状态. 而 2-2 结构 却由于外延磁性层受基片的完全约束作用而使其磁 电耦合作用变得微弱, 导致磁场诱导电极化值很小.

$\mathrm{Nan}$ 等人还对体积分数对磁电耦合的影响进行 了计算, 对于复合薄膜, 其有效自发极化强度随磁致 伸缩相的增加而减小(图 18(a)), 1-3 结构薄膜与 2-2 结 构薄膜也表现出较大差别. 随着 $\mathrm{CFO}$ 含量从 0 增加到 1, MIEP呈先增加后减小变化趋势(图 18(b)), 而且 1-3 结构变化显著而 2-2 结构变化很微弱. 随后, Liu等人 [57,58]利用改进的Landau-Ginsburg-Devonshire热力学 理论也计算了不同结构的 $\mathrm{BaTiO}_{3} / \mathrm{CoFe}_{2} \mathrm{O}_{4}$ 纳米复合 薄膜的磁-电效应, 得到了类似的结论.

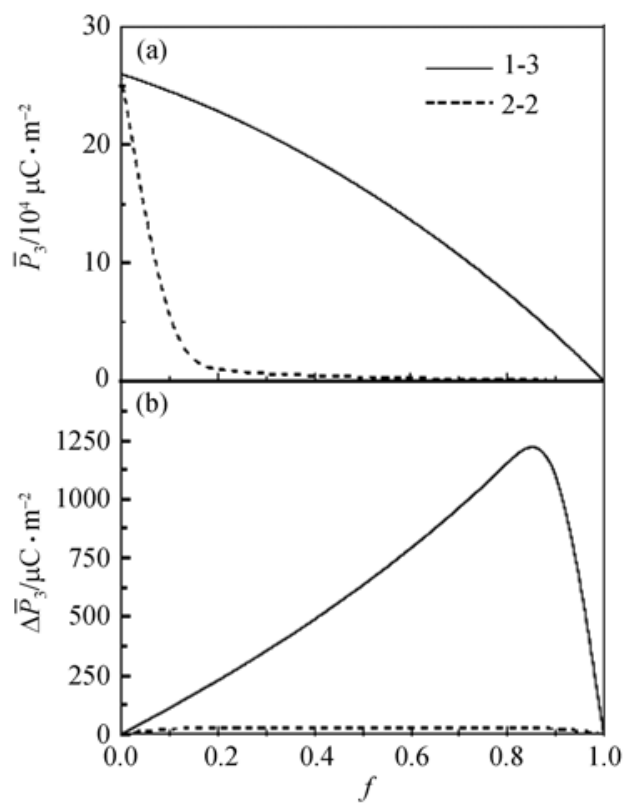

图18 对于 1-3, 2-2 两种结构薄膜, 在磁场强度为 $250 \mathrm{kA} / \mathrm{m}$ 时极化强度(a)和磁场诱导极化强度 (b)随磁致伸缩相的体 积分数的变化情况 ${ }^{[56]}$

最近, 宾夕法尼亚州立大学的Zhang等人 ${ }^{[59]}$ 用相 场方法对 1-3 型纳米复合薄膜中的磁电耦合效应也进 行了计算, 模拟了薄膜厚度、形貌以及基片约束等因 素对磁场诱导电极化的影响. 这种模型通过三个场 来描述任意的一种显微状态: 局域的磁化场、局域的 
极化场和描述复合薄膜中各相空间分布的有序参量 场. 对 1-3 结构的 $\mathrm{BaTiO}_{3}-\mathrm{CoFe}_{2} \mathrm{O}_{4}$ 纳米复合薄膜的模 拟计算表明, 磁场诱导电极化受薄膜厚度以及基片 约束作用的影响较大, 如图 19 所示. 计算的MIEP与 格林函数方法结果相近.

以上的计算和模拟都是基于连续介质方法, 模 拟由磁致伸缩效应和压电效应通过应力/应变作用的 传递耦合而产生的乘积磁电效应. 近来, Duan等人 ${ }^{[60]}$ 利用第一性原理计算讨论了一种由界面结合的铁电 性和磁性耦合而产生的磁电效应。他们对 $\mathrm{Fe} / \mathrm{BaTiO}_{3}$ 超晶格模型进行了计算 ${ }^{[600]}$, 如图 20 所示, 取中间 $\mathrm{TiO}_{2}$ 层为参考面, 则该结构是上下镜面对称的. 在顺电态, 界面处的磁矩也完全对称. 但是计算结果表明, 该结 构的磁矩分布已经与块体有了较大区别. 例如, 界面 处的 $\mathrm{Fe}$ 原子的磁矩已经由块体中的 $2.20 \mu_{\mathrm{B}}$ 增加到 $2.64 \mu_{\mathrm{B}}, \mathrm{Fe} / \mathrm{BaTiO}_{3}$ 界面处的耦合以及电荷交换导致 了界面 $\mathrm{Fe}$ 原子磁矩的增加, 同时 $\mathrm{Ti}$ 和 $\mathrm{O}$ 原子也由于电 荷交换产生了诱导磁矩, $\mathrm{O}$ 原子存在 $0.05 \mu_{\mathrm{B}}$ 的与 $\mathrm{Fe}$ 原 子磁矩平行的诱导磁矩, 而 $\mathrm{Ti}$ 原子存在 $-0.30 \mu_{\mathrm{B}}$ 的与 $\mathrm{Fe}$ 原子磁矩反平行的诱导磁矩. 但是在外加电场时, $\mathrm{BaTiO}_{3}$ 被极化, $\mathrm{BaTiO}_{3}$ 中出现铁电位移, 特别是 $\mathrm{Ti}$ 原 子的位移，打破了上下两个界面以中间 $\mathrm{TiO}_{2}$ 层为参 考面的对称状态, 从而使得上下两个界面处的原子 磁矩发生变化. 对于下界面, 铁电极化方向偏离 $\mathrm{Fe}$ 原 子, 计算结果表明, $\mathrm{Fe}$ 原子磁矩增强到 $2.67 \mu_{\mathrm{B}}$, Ti原 子磁矩变化到 $-0.18 \mu_{\mathrm{B}}$; 对应的在上界面, 铁电极化 方向偏向 $\mathrm{Fe}$ 原子, $\mathrm{Fe}$ 原子磁矩减小到 $2.61 \mu_{\mathrm{B}}$, Ti原子 磁矩变化到 $-0.40 \mu_{\mathrm{B}}$. 于是, 在这两个界面之间的原 子磁矩产生一个差值: $\Delta \mu_{\mathrm{Fe}}=0.06 \mu_{\mathrm{B}}, \Delta \mu_{\mathrm{T}}=0.22 \mu_{\mathrm{B}}$. 从 而改变了界面处的磁化, 使得这种结构实现了电性

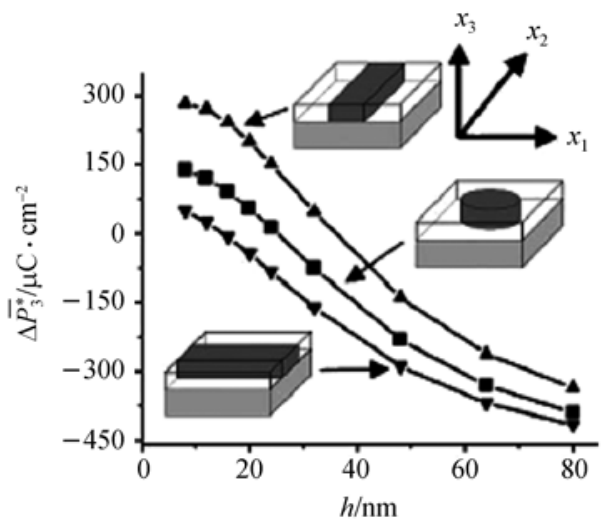

能和磁性能之间的耦合，也即产生了磁电效应。

根据上述计算结果对该磁电效应产生的磁电系 数按照 $\alpha=\mu \cdot \Delta M / E_{\mathrm{c}}$ 进行估算, 结果得到的磁电系 数约为 $\alpha \approx 0.01 \mathrm{G} \cdot \mathrm{cm} / \mathrm{V}$, 这与Zavaliche等人 ${ }^{[30]}$ 报道 的 1-3 柱状结构的 $\mathrm{BiFeO}_{3} / \mathrm{CoFe}_{2} \mathrm{O}_{4}$ 磁电复合薄膜中的 磁电系数相当, 表明这种由界面键合产生的磁电效 应与由应变耦合产生的磁电效应是可比拟的. 随后, Yamauchi等人 ${ }^{[61]}$ 在 $\mathrm{Co}_{2} \mathrm{MnSi} / \mathrm{BaTiO}_{3}$ 体系中用类似的 计算方法也得到了类似的结果. 这种机制产生的磁 电效应目前还只是理论上的计算, 实验观察还有待 证实. 从本质上讲, 这种机制与前面讨论的应力/应 变传递机制是类似的: 在复合体系中, 耦合磁电效应 来源于跨越压电/铁电与磁性界面的某种应变传递耦 合作用. 但按照这种理论模型, 叠层薄膜中的磁电耦 合将不受基片的应力约束, 如果这种效应能够在实 验中得到证实, 将对磁电薄膜材料, 特别是叠层结构 的薄膜材料有重要意义.

\section{3 结语}

如今, 压电/铁电材料和铁磁材料已分别得到了 实际的应用, 而多铁性材料同时具有铁电和铁磁性, 此外由于耦合还会产生磁电效应，在继承两者优点 的同时提高了器件设计的自由度, 有利于器件向小 型化和多功能化方向发展, 必然具有更广阔的应用 空间. 目前, 块体磁电复合材料已经有了初步的应用, 在传感器、换能器等方向都已经有了器件原型. 近几 年, 磁电纳米结构已成为人们日益关注的热点. 这种 纳米结构, 特别是磁电薄膜易于与芯片集成, 这是与 现有微电子器件兼容的一个必要条件, 因此磁电纳 米结构及其器件具有更大的潜力. 但是, 目前磁电纳

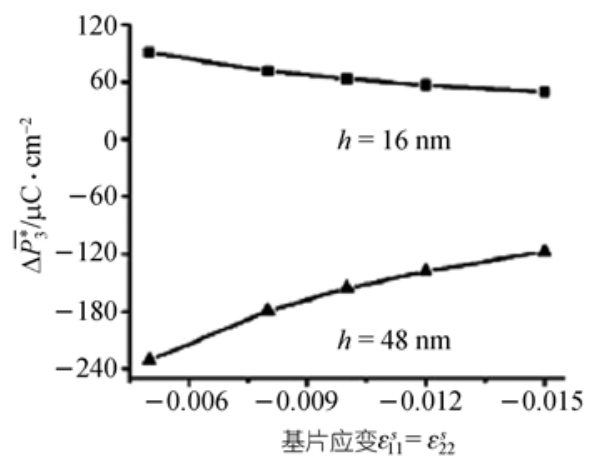

图19复合薄膜的磁场诱导电极化随薄膜厚度(a)以及基片应变(b)的变化关系 ${ }^{[59]}$ 


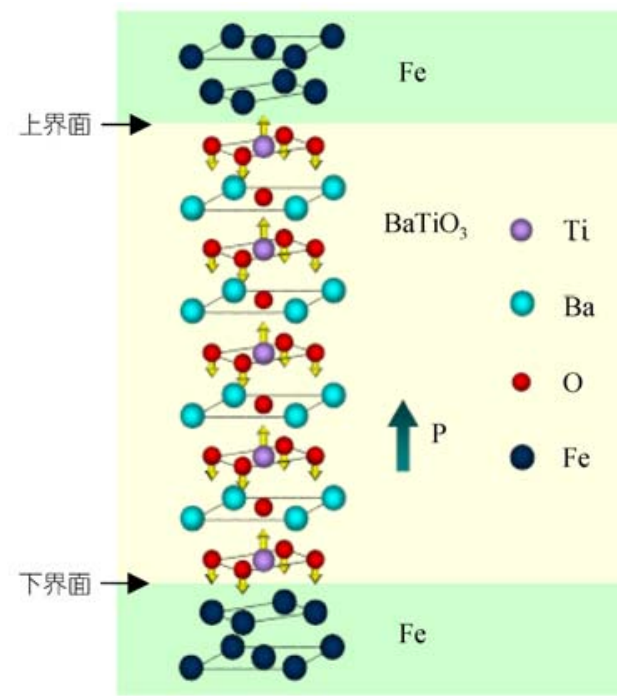

图20 $\mathrm{Fe}\left(\mathrm{BaTiO}_{3}\right)_{4} \mathrm{Fe}$ 原子结构示意图 箭头标示了原子位移方向

米结构还仅仅处于初级阶段，我们必须首先要克服 以下两个基础性挑战.

第一个问题是物理机制方面: 我们怎样通过一 些切实可行的、适用于现实中的磁电材料和器件的方 法, 来对磁电纳米结构中每个重要原子的特性、位置 和作用进行调控? 在纳米结构自组装过程中, 通过 精确控制成分、原子排布和界面来控制我们想要的磁
电纳米结构的生长, 这是很有意义的, 但也是一个难 题. 实际上, 可控制的自组装工艺目前已经取得了一 些进展, 也已成为控制生长纳米材料和纳米器件的 重要途径. 例如, 长程有序的柱状自组装纳米结构, 毫无疑问将在多铁性领域和光子应用领域产生巨大 的影响. 高质量的叠层异质结构, 例如磁电超晶格, 也非常有望成为一种新的多铁性结构和器件.

第二个问题是概念方面: 我们怎么理解这样一 种纳米磁电复合系统, 一方面它太大而不能完全用 强关联体系来处理, 但如果用静态的连续介质方法来 处理又显得太小. 关于纳米结构中的磁电耦合作用还 有很多没有解决的问题, 例如, 耦合作用的尺度有多 大? 磁性随时间的变化与铁电反转有什么关系? 能导 致磁畴完全翻转的磁场最小值究竟有多小? 纳米磁电 结构的性能与化学工艺有什么关系? 本质上耦合作用 消失或者改变是否存在一个临界尺寸?

多铁性磁电复合薄膜相比于块体磁电复合材料, 更有希望应用于微传感器和多态存储等领域, 由于 与微电子器件和工艺的兼容而使得薄膜具有更广阔 的应用前景. 同时, 磁电复合薄膜的研究刚起步, 存 在很多尚未解决的问题, 更需要投入更多的关注, 付 出更多的努力, 以进一步推动磁电材料研究的快速 发展.

\section{参考文献}

1 Eerenstein W, Mathur N D, Scott J F. Multiferroic and magnetoelectric materials. Nature, 2006, 442: 759-765[doi]

2 Fiebig M. Revival of the magnetoelectric effect. J Phys D: Appl Phys, 2005, 38: R123 - R152[doi]

3 Ramesh R, Spaldin N A. Multiferroics: Progress and prospects in thin films. Nature Mater, 2007, 6: 21-29 [doi]

4 Curie P. Sur la Symétrie dans les Phénomènes Physiques. J Phys, 1894, 3: 393

5 Rado G T, Folen V J. Observation of the magnetically induced magnetoelectric effect and evidence for antiferromagnetic domains. Phys Rev B, 1961, 7(8): 310[doi]

6 Folen V J, Rado G T, Stalder E W. Anisotropy of the magnetoelectric effect in $\mathrm{Cr}_{2} \mathrm{O}_{3}$. Phys Rev Lett, 1961, 6: 607-609[doi]

7 Schmid H, Janner A, Grimmer H, et al. Proceedings of the 2nd International Conference on Magnetoelectric Interaction Phenomena in Crystals MEIPIC-2. Ferroelctrics, 1994, 161\&162

8 Bichurin M. Proceedings of the 3rd International Conference on Magnetoelectric Interaction Phenomena in Crystals MEIPIC-3. Ferroelctrics, 1997, 204

9 Bichurin M. Proceedings of the 4th International Conference of Magnetoelectric Interaction Phenomena in Crystals MEIPIC-4. Ferroelectrics, 2002, 279 \& 280

10 Hur N, Park S, Sharma P A, et al. Colossal magnetodielectric effects in DyMn $\mathrm{O}_{5}$. Phys Rev Lett, 2004, 93(10): 107207[doi]

11 Wang J, Neaton J B, Zheng H, et al. Epitaxial $\mathrm{BiFeO}_{3}$ multiferroic thin film heterostructures. Science, 2003, 299(5613): 1719$1722 \underline{\text { doi] }}$

12 Wang Y, Jiang Q H, He H C, et al. Multiferroic $\mathrm{BiFeO}_{3}$ thin films prepared via a simple sol-gel method. Appl Phys Lett, 2006, 88(14): $142503 \underline{\text { [doi] }}$ 
13 Jiang Q H, Nan C W, Shen Z J. Synthesis and properties of multiferroic La-modified BiFeO 3 ceramics. J Am Ceram Soc, 2006, 89(7): 2123-2127

Suchtelen J V. Product properties: A new application of composite materials. Philips Rep, 1972, 27: 28 - 37

Nan C W. Magnetoelectric effect in composites of piezoelectric and piezomagnetic phases. Phys Rev B, 1994, 50: 6082 - 6088 [doi]

Van Run A M J, Terrell D R, Scholing J H. An in situ grown eutectic magnetoelectric composite material. Part II: Physical properties. J Mater Sci, 1974, 9: 1710-1714 $\underline{\text { [doi] }}$

Boomgaard V D, Born R A G. A sintered magnetoelectric composite materials $\mathrm{BaTiO}_{3}-\mathrm{Ni}(\mathrm{Co}, \mathrm{Mn}) \mathrm{Fe}_{2} \mathrm{O}_{4}$. J Mater Sci, 1978, $13: 1538$ -1548[doi]

Boomgaard V D, Van Run J, Van Suchtelen A M T G J. Piezoelectric-piezomagnetic composites with magnetoelectric effect. Ferroelectrics, 1976, 14: 728-728

19 Zhai J Y, Cai N, Shi Z, et al. Coupled magnetodielectric properties of laminated $\mathrm{PbZr}_{0.53} \mathrm{Ti}_{0.47} \mathrm{O}_{3} / \mathrm{NiFe}_{2} \mathrm{O}_{4}$ ceramics. J Appl Phys, 2004, 95(10): 5685-5690[doi]

20 Zhai J Y, Cai N, Shi Z, et al. Magnetic-dielectric properties of $\mathrm{NiFe}_{2} \mathrm{O}_{4}$ /PZT particulate composites. J Phys D: Appl Phys, 2004, 37(6): $823-827$ [doi]

21 Ryu J H, Priya S, Carazo A V, et al. Effect of the magnetostrictive layer on magnetoelectric properties in lead zirconate titanate/terfenol-D laminate composites. J Am Ceram Soc, 2001, 84: 2905-2908

22 Dong S X, Zhai J Y, Li J F, et al. Magnetoelectric effect in Terfenol-D/Pb(Zr,Ti) $\mathrm{O}_{3} /$ mu-metal laminate composites. Appl Phys Lett, 2006, 89(12): 122903 [doi]

23 Dong S X, Chen J R, Li J F, et al. Enhanced magnetoelectric effects in laminate composites of Terfenol-D/Pb( $\mathrm{Zr}, \mathrm{Ti}) \mathrm{O}_{3}$ under resonant drive. Appl Phys Lett, 2003, 83: 4812 [1doi]

24 Nan C W, Liu L, Cai N, et al. A three-phase magnetoelectric composite of piezoelectric ceramics, rare-earth iron alloys, and polymer. Appl Phys Lett, 2002, 81: 3831-3833[doi]

25 Shi Z, Nan C W, Zhang J, et al. Magnetoelectric effect of $\mathrm{Pb}(\mathrm{Zr}, \mathrm{Ti}) \mathrm{O}_{3}$ rod arrays in a (Tb,Dy)Fe 2 /epoxy medium. Appl Phys Lett, 2005, 87: 012503 [doi]

Cai N, Nan C W, Zhai J Y, et al. Large high-frequency magnetoelectric response in laminated composites of piezoelectric ceramics, rare-earth iron alloys and polymer. Appl Phys Lett, 2004, 84: 3516 - 3518[doi]

27 Wan J G, Liu J M, Chan H L W, et al. Giant magnetoelectric effect of a hybrid of magnetostrictive and piezoelectric composites. J Appl Phys, 2003, 93(12): 9916-9919 [doi]

Zheng H, Wang J, Lofland S E, et al. Multiferroic $\mathrm{BaTiO}_{3}-\mathrm{CoFe}_{2} \mathrm{O}_{4}$ nanostructures. Science, 2004, 303: 661-663 [doi]

Zheng H, Wang J, Mohaddes-Ardabili L, et al. Three-dimensional heteroepitaxy in self-assembled $\mathrm{BaTiO}_{3}-\mathrm{CoFe}_{2} \mathrm{O}_{4}$ nanostructures. Appl Phys Lett, 2004, 85: 2035-2037 [doi]

Zavaliche F, Zheng H, Mohaddes-Ardabili L, et al. Electric field-induced magnetization switching in epitaxial columnar nanostructures. Nano Lett, 2005, 5: 1793-1796 [doi]

31 Zheng H, Zhan Q, Zavaliche F, et al. Controlling self-assembled perovskite-spinel nanostructures. Nano Lett, 2006, 6: 1401$1407 \underline{\text { [doi] }}$

32 Zheng $\mathrm{H}$, Straub F, Zhan Q, et al. Self-assembled growth of $\mathrm{BiFeO}_{3}-\mathrm{CoFe}_{2} \mathrm{O}_{4}$ nanostructures. Adv Mater, 2006, 18: 2747-2752[doi]

33 Levin I, Li J, Slutsker J, et al. Design of self-assembled multiferroic nanostructures in epitaxial films. Adv Mater, 2006, 18: 20442047 [doi]

Wan J G, Wang X W, Wu Y J, et al. Magnetoelectric $\mathrm{CoFe}_{2} \mathrm{O}_{4}-\mathrm{Pb}\left(\mathrm{Zr}\right.$, Ti) $\mathrm{O}_{3}$ composite thin films derived by a sol-gel process. Appl Phys Lett, 2005, 86: 122501 [doi]

Zhong X L, Wang J B, Liao M, et al. Multiferroic nanoparticulate $\mathrm{Bi}_{3.15} \mathrm{Nd}_{0.85} \mathrm{Ti}_{3} \mathrm{O}_{12}-\mathrm{CoFe}_{2} \mathrm{O}_{4}$ composite thin films prepared by a chemical solution deposition technique. Appl Phys Lett, 2007, 90: 152903 [doi]

36 Ryu H, Murugavel P, Lee J H, et al. Magnetoelectric effects of nanoparticulate $\mathrm{Pb}\left(\mathrm{Zr}_{0.52} \mathrm{Ti}_{0.48}\right) \mathrm{O}_{3}-\mathrm{NiFe}_{2} \mathrm{O}_{4}$ composite films. Appl Phys Lett, 2006, 89: 102907[doi]

Zhai J, Cai N, Shi Z, et al. Magnetic-dielectric properties of $\mathrm{NiFe}_{2} \mathrm{O}_{4} / \mathrm{PZT}$ particulate composites. J Phys D, 2004, 37: 823-827[doi]

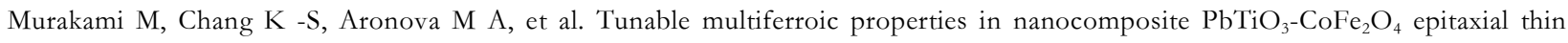
films. Appl Phys Lett, 2005, 87: 112901 [10i] 
40 Chang K -S, Aronova M A, Lin C -L, et al. Exploration of artificial multiferroic thin-film heterostructures using composition spreads. Appl Phys Lett, 2004, 84: 3901-3903

41 He H C, Wang J, Zhou J P, et al. Ferroelectric and ferromagnetic behavior of $\mathrm{Pb}\left(\mathrm{Zr}_{0.52} \mathrm{Ti}_{0.48}\right) \mathrm{O}_{3}-\mathrm{Co}_{0.9} \mathrm{Zn}_{0.1} \mathrm{Fe}_{2} \mathrm{O}_{4}$ multilayered thin films prepared via solution processing. Adv Funct Mater, 2007, 17: 1333-1338 [doi]

42 He H C, Zhou J P, Wang J, et al. Multiferroic $\mathrm{Pb}\left(\mathrm{Zr}_{0.52} \mathrm{Ti}_{0.48}\right) \mathrm{O}_{3}-\mathrm{Co}_{0.9} \mathrm{Zn}_{0.1} \mathrm{Fe}_{2} \mathrm{O}_{4}$ bilayer thin films via a solution processing. Appl Phys Lett, 2006, 89: 052904 $\underline{\text { [doi] }}$

43 Deng C, Zhang Y, Ma J, et al. Magnetic-electric properties of epitaxial multiferroic $\mathrm{NiFe}_{2} \mathrm{O}_{4}-\mathrm{BaTiO}_{3}$ heterostructure. J Appl Phys, 2007, 102: 074114 [doi]

Zhou J P, He H C, Zhang Y, et al. Electric and magnetic properties of $\mathrm{CoFe}_{2} \mathrm{O}_{4} / \mathrm{Pb}\left(\mathrm{Zr}_{0.52} \mathrm{Ti}_{0.48}\right) \mathrm{O}_{3}$ bilayer thin films prepared by pulsed-laser deposition. Appl Phys A, 2007, 89: 553-558 [doi]

Zhou J P, He H C, Shi Z, et al. Magnetoelectric $\mathrm{CoFe}_{2} \mathrm{O}_{4} / \mathrm{Pb}\left(\mathrm{Zr}_{0.52} \mathrm{Ti}_{0.48}\right) \mathrm{O}_{3}$ double-layer thin film prepared by pulsed-laser deposition. Appl Phys Lett, 2006, 88: 013111 [10i]

46 Ryu S, Park J H, Jang H M. Magnetoelectric coupling of [00l]-oriented $\mathrm{Pb}\left(\mathrm{Zr}_{0.4} \mathrm{Ti}_{0.6}\right) \mathrm{O}_{3}-\mathrm{Ni}_{0.8} \mathrm{Zn}_{0.2} \mathrm{Fe}_{2} \mathrm{O}_{4}$ multilayered thin films. Appl Phys Lett, 2007, 91: 142910 [doi]

47 Ziese M, Bollero A, Panagiotopoulos I, et al. Magnetoresistance switch effect in a multiferroic $\mathrm{Fe}_{3} \mathrm{O}_{4} / \mathrm{BaTiO}_{3}$ bilayer. Appl Phys Lett, 2006, 88: 212502[doi]

Murugavel P, Saurel D, Prellier W, et al. Enhanced magnetoresistance in ferromagnetic $\mathrm{Pr}_{0.85} \mathrm{Ca}_{0.15} \mathrm{MnO}_{3} /$ ferroelectric $\mathrm{Ba}_{0.6} \mathrm{Sr}_{0.4} \mathrm{TiO}_{3}$ superlattice films. Appl Phys Lett, 2004, 85: 4424-4426 [doi]

49 Murugavel P, Singh M P, Prellier W, et al. The role of ferroelectric-ferromagnetic layers on the properties of superlattice-based multiferroics. J Appl Phys, 2005, 97: 103914 [doi]

50 Singh M P, Prellier W, Simon Ch, et al. Magnetocapacitance effect in perovskite-superlattice based multiferroics. Appl Phys Lett, 2005, 87: 022505 [doi]

51 Singh M P, Prellier W, Mechin L, et al. Effect of ferroelectric layers on the magnetocapacitance properties of superlattices-based oxide multiferroics. Appl Phys Lett, 2006, 88: 012903 [doi]

Stein S, Wuttig M, Viehland D, et al. Magnetoelectric effect in sputtered composites. J Appl Phys, 2005, 97: 10Q301 [doi]

Eerenstein W, Wiora M, Prieto J L, et al. Giant sharp and persistent converse magnetoelectric effects in multiferroic epitaxial heterostructures. Nature Mater, 2007, 6: 348-351 [doi]

Wu T, Zurbuchen M A, Saha S, et al. Observation of magnetoelectric effect in epitaxial ferroelectric film/manganite crystal heterostructures. Phys Rev B, 2006, 73: 134416[doi]

Zurbuchen M A, Wu T, Saha S, et al. Multiferroic composite ferroelectric-ferromagnetic films. Appl Phys Lett, 2005, 87: 232908[doi]

Nan C W, Liu G, Lin Y -H, et al. Magnetic-field-induced electric polarization in multiferroic nanostructures. Phys Rev Lett, 2005, 94: 197203 [doi]

Liu G, Nan C W, Xu Z K, et al. Coupling interaction in multiferroic $\mathrm{BaTiO}_{3}-\mathrm{CoFe}_{2} \mathrm{O}_{4}$ nanostructures. J Phys D: Appl Phys, 2005, 38: $2321-2326 \underline{\text { doi] }}$

58 Liu G, Nan C W, Sun J. Coupling interaction in nanostructured piezoelectric/magnetostrictive multiferroic complex films. Acta Mater, 2006, 54: 917-925[doi]

59 Zhang J X, Li Y L, Schlom D G, et al. Phase-field model for epitaxial ferroelectric and magnetic nanocomposite thin films. Appl Phys Lett, 2007, 90: 052909[doi]

60 Duan C -G, Jaswal S S, Tsymbal E Y. Predicted magnetoelectric effect in $\mathrm{Fe} / \mathrm{BaTiO}_{3}$ multilayers: ferroelectric control of magnetism. Phys Rev Lett, 2006, 97: 047201 [doi]

61 Yamauchi K, Sanyal B, Picozzi S. Interface effects at a half-metal/ferroelectric junction. Appl Phys Lett, 2007, 91: 062506 [doi] 